\title{
Condensate splitting in an asymmetric double well for atom chip based sensors
}

\author{
B. V. Hall * S. Whitlock, R. Anderson, P. Hannaford, and A. I. Sidorov \\ ARC Centre of Excellence for Quantum-Atom Optics and \\ Centre for Atom Optics and Ultrafast Spectroscopy, \\ Swinburne University of Technology, Hawthorn, Victoria 3122, Australia
}

(Dated: October 22, 2018)

\begin{abstract}
We report on the adiabatic splitting of a $\mathrm{BEC}$ of ${ }^{87} \mathrm{Rb}$ atoms by an asymmetric double-well potential located above the edge of a perpendicularly magnetized TbGdFeCo film atom chip. By controlling the barrier height and double-well asymmetry the sensitivity of the axial splitting process is investigated through observation of the fractional atom distribution between the left and right wells. This process constitutes a novel sensor for which we infer a single shot sensitivity to gravity fields of $\delta g / g \approx 2 \times 10^{-4}$. From a simple analytic model we propose improvements to chip-based gravity detectors using this demonstrated methodology.
\end{abstract}

PACS numbers: 03.75.Be, 03.75.Kk, 39.25.+k, 03.75.Nt, 39.90.+d

Atom interferometry techniques have been successfully applied to the precision sensing of inertial forces, with demonstrated measurement of the Earth's rotation rate and the acceleration due to the Earth's gravity [1, 2]. More recently with the advent of atom chips physicists are seeking to exploit atom interferometric methods using trapped samples of ultra-cold atoms and Bose-Einstein condensates (BEC) 3, 4]. While these trapped atom interferometers are in their infancy, a number of pioneering experiments have already utilized the inherent low energy scale of ultra-cold atoms and BECs as probes of external phenomena. Such experiments include using BECs/ultra-cold atoms to probe the magnetic potential landscape above conducting micro-wires [5] and magnetic films [6]; measuring the electric field distribution on charged micro-wires [7]; quantifying the amount of near-field radiation due to Johnson noise in conductive and dielectric surfaces [ $[$, [9]; and using the dynamical behavior of a condensate to precisely measure the CasimirPolder force as a function of distance 10. In addition trapped bosonic ${ }^{88} \mathrm{Sr}$ atoms at nano-Kelvin temperatures have recently been used to measure gravity with a estimated sensitivity of $\delta g / g \approx 4 \times 10^{-5}[11$.

In this Letter we report on a novel sensor which utilizes adiabatic axial splitting of a BEC in an asymmetric double well. This potential is created above a perpendicularly magnetized TbGdFeCo film [12] which has previously been used to study the influence of magnetic film inhomogeneity on ultra-cold atoms 6]. We show that while cloud fragmentation was initially reported as a deleterious effect for atom chips 13, 14], our unique potential landscape can be utilized to realize a precision sensor which measures potential gradients. Time dependent magnetic fields in combination with pulsed radio frequency (rf) spectroscopy and absorption imagining are used to characterize the double-well separation $\lambda$, the barrier height $\beta$ and the asymmetry $\Delta$ as a function of trap-surface distance. Results are then presented for adiabatic BEC splitting experiments where the frac-

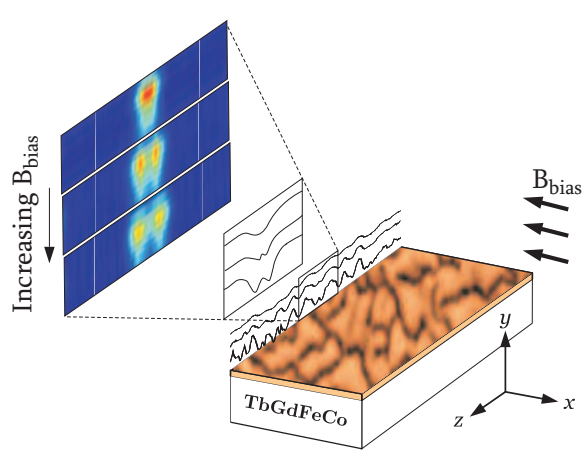

FIG. 1: (Color online) Schematic showing the corrugated potential above the edge of the TbGdFeCo film. A double well can be used to axially split a BEC by increasing $\mathrm{B}_{\text {bias }}$. The corrugated potential is due to the presence of inhomogeneity in the magnetic film.

tional atom number distribution is used as a measure of the double-well asymmetry to much higher precision than that obtained with rf spectroscopy. A simple analytic model is presented to determine the sensitivity of this technique and its dependence on atom number, trap parameters and atomic properties. Finally we use this model to propose improvements to atom chip sensors based on this method.

A detailed description of the apparatus, including experiments performed with a BEC on a perpendicularly magnetized film atom chip, have been described elsewhere [6, 12]. In brief, our atom chip consists of a uniformly magnetized TbGdFeCo film on a $0.3 \mathrm{~mm}$ thick glass slide which is epoxied to a machined Ag foil structure. The Ag structure allows currents to be passed in U- and Z-shape paths for surface magneto-optical trap (MOT) and Ioffe-Pritchard trap geometries [15]. A uniform magnetic field $B_{\text {bias }}$ combines with the magnetic field above the film edge to provide radial confinement while a pair of end wires provide axial confinement. A small amount of inhomogeneity in the TbGdFeCo film results in a corrugation of the axial potential with a pe- 


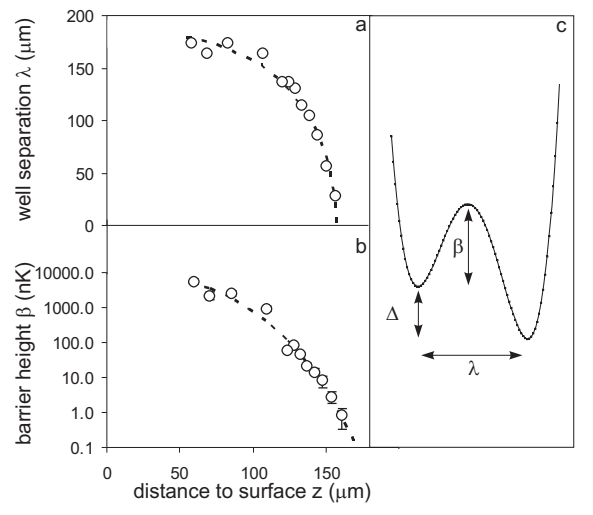

FIG. 2: Characterization of the double well as a function of trap-surface separation is performed using two component clouds. The dashed line in (a) and (b) are to guide the eye. The well separation $\lambda$, barrier height $\beta$ and trap asymmetry $\Delta$ are shown schematically in (c).

riod of about $390 \mu \mathrm{m}$. This corrugating potential introduces fragmentation to the ultra-cold atom cloud which becomes more pronounced as the cloud approaches the surface. The measured potential is depicted in Fig. 1 along with a schematic of the region of interest, where the double well is located with its center at $z=0$. This double well arises from higher frequency components of the magnetization inhomogeneity exerting a larger influence as the film trap is brought closer to the surface with increasing $B_{\text {bias }}$ in the x-direction.

Since the magnetic topography is created by random magnetic inhomogeneities present in the film, a systematic characterization of the double-well system is required. To achieve this a partially condensed cloud of $10^{5}$ ${ }^{87} \mathrm{Rb}$ atoms in the $F=2, m_{F}=2$ state is first prepared in a single-well potential located $170 \mu \mathrm{m}$ from the surface. Over $500 \mathrm{~ms} B_{\text {bias }}$ is ramped to the desired value at which point the trap is rapidly switched off. The spatial distribution is determined via absorption imaging after a $2 \mathrm{~ms}$ expansion time. The potential is then mapped by fitting standard Thomas-Fermi/Gaussian distribution functions which are scaled by the cloud temperature and chemical potential $\mu$ [5]. From these measurements the separation $\lambda$ and the barrier height $\beta$ of the double-well system are determined for a range of trap heights, (Fig. (2). Faster splitting times are also used to induce dipole mode oscillations in the condensate to measure both the axial and radial trap frequencies [16]. Remarkably, the left and right wells have trapping frequencies which are identical to within a few percent.

Another parameter of interest depicted in Fig. 2. is the asymmetry $\Delta$ between the left and right wells. A nonzero $\Delta$ results in a marked lop-sidedness in condensate distribution for splitting experiments when the barrier height is smaller than the chemical potential $(\beta<\mu)$. Similar observations involving the radial splitting of a

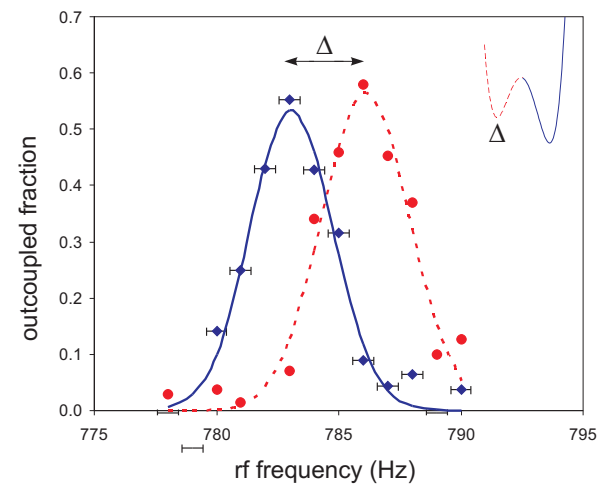

FIG. 3: (Color online) Results of pulsed radio frequency spectroscopy, performed on a condensate which has been split symmetrically and then exposed to a large asymmetry. The shift in the output coupled spectra yields a measure of $\Delta$.

thermal cloud in a double-well potential have been reported previously [17]. Prior to this investigation the apparatus was mounted on a floating vibration-isolating optical table. Systematic drifts in the tilt of the chip were observed throughout the day due to changes in line pressure feeding the isolating legs. Operating the table as a rigid structure eliminated this effect; however a slight tilt of the atom chip with respect to gravity still remains for identical end wire currents. This gravitational gradient is canceled in the above experiments by applying a small magnetic field gradient $\frac{\partial B_{z}}{\partial z}$ which is controlled by an end wire current imbalance $\pm \delta I$. For two infinitely thin parallel wires propagating current in the $\widehat{x}$ direction, separated by a distance $d$, the gradient along $z$ at height $y$ above the midpoint between these wires is given by

$$
\frac{\partial B_{z}}{\partial z}=\frac{8 \mu_{0} \delta I}{\pi} \frac{d y}{\left(d^{2}+4 y^{2}\right)^{2}} \quad T m^{-1}
$$

Equation 1illustrates that for a fixed height and separation the magnetic field gradient is proportional to $\delta I$. To circumvent finite size effects and imbalances in the machined end wire uniformity a method of calibrating $\Delta$ in situ was developed. This entails adiabatically splitting a condensate in a symmetric double well with barrier height $\beta>\mu$ such that no spillage can occur between the left and right wells. A large asymmetry is then applied over $500 \mathrm{~ms}$ by tuning the end wire current imbalance $\delta I$. Next a low intensity $40 \mu$ s pulse of fixed frequency rf radiation (Rabi frequency $\Omega / 2 \pi=0.43 \mathrm{kHz}$ ) is introduced. This outcouples atoms from either well in a magnetic energy selective manner $\left(\mathrm{h} \nu=g_{F} m_{F} \mu_{B}|B|\right)$ and this outcoupled fraction is measured by absorption imaging. Figure 3 shows the results of this spectroscopic technique where the separation of the left/right well distributions is a measure of the asymmetry $\Delta$. This calibration process was repeated for various $\delta I$ values yielding $\Delta=2.0(1) \mathrm{Hz} / \mathrm{mA}$. 


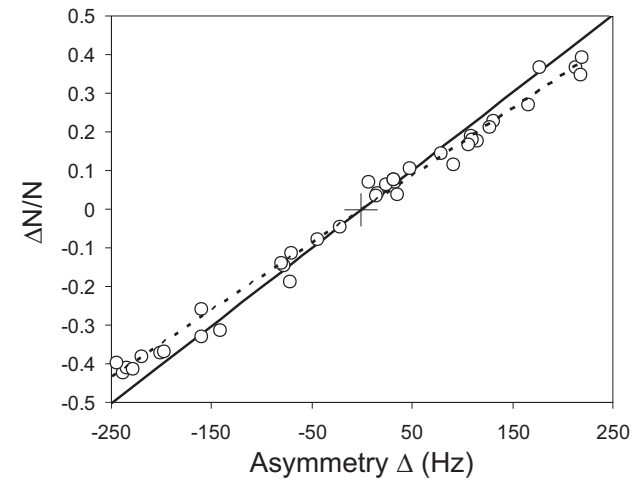

FIG. 4: Fractional number difference versus the double-well potential asymmetry. The asymmetry is varied by randomly changing the end wire current imbalance $\delta I$. The experimental data points (open circles) and the line of best fit (dashed line) compare well with the simple analytic result (solid line).

We now focus on the dynamical splitting of a condensate in a double well with asymmetry. To remain in the ground state of the potential the splitting time $t_{s}$ must be greater than $\Delta^{-1}$ thus ensuring adiabaticity of the splitting process. This condition establishes a lower bound for the resolvable asymmetry of this method which can be related to the following experimental limitations on $t_{s}$. The longest splitting time is determined by background gas collisions $\left(\tau_{R b} \approx 100 \mathrm{~s}\right)$ which decrease the sensitivity of the measurement by reducing the signal to noise ratio by $e^{\frac{-t_{s}}{2 \tau_{R b}}}$. A more relevant limitation is the condensate lifetime $\tau_{C}$ which can range from $20 \mathrm{~ms}$ to several seconds. Processes which shorten $\tau_{C}$ above atom chips include spin flip loss near surfaces [18], parametric heating due to technical noise [19], density dependent collisional loss and heating mechanisms [20].

The magnetic trapping potential generated by the TbGdFeCo film is well suited for minimizing these limitations. The double well exists far from the chip surface $(170 \mu \mathrm{m})$ which, in conjunction with the reduced thickness of the TbGdFeCo-Cr-Au material $(1 \mu \mathrm{m})$, significantly lowers the spin-flip loss rate. In addition, due to the nature of the perpendicularly magnetized film, technical noise due to current fluctuations are not present on the structure that generates the corrugated potential. Moreover the radial and axial trapping potentials are relatively weak and the number of condensed atoms is kept low to avoid high density loss and heating processes. With these conditions we have measured heating rates for small thermal clouds in single-well magnetic film traps as low as $3 \mathrm{nK} / \mathrm{s}$ and condensate lifetimes of $5 \mathrm{~s}$ have been observed without the application of a radiofrequency shield. In future experiments this lifetime may be further improved by preparing the condensate in the $\left(F=1, m_{F}=-1\right)$ ground state.

With $\Delta$ calibrated the sensitivity of the splitting pro- cess for small values of $\Delta$ and a large splitting time $t_{s}$ is examined. A small condensate of $4.6 \times 10^{4}$ atoms is prepared in a single well $170 \mu \mathrm{m}$ from the film surface. $B_{\text {bias }}$ is ramped between 0.130 and $0.135 \mu \mathrm{T}$ over $2 \mathrm{~s}$, producing a tailored double well $155 \mu \mathrm{m}$ from the surface with a well separation $\lambda \approx 70 \mu \mathrm{m}$. At this position $\beta \approx \frac{1}{4} \mu$ and the chemical potential of the right $(\mu)$ and left $\left(\mu^{\prime}\right)$ wells is given by $\mu=\mu^{\prime}+\Delta$. The asymmetry is set by the end wire current imbalance which ranges between $\pm 117 \mathrm{~mA}$ over the course of these experiments. At the end of the adiabatic splitting process $B_{\text {bias }}$ is rapidly increased over $10 \mathrm{~ms}$ to further separate each well thus enhancing the spatial discrimination of the imaging process. All trapping fields are then turned off and each cloud expanded for $2 \mathrm{~ms}$ prior to taking an absorption image. A CCD camera captures the image with a resolution of $3.5 \mu \mathrm{m}$ per pixel. This experimental cycle takes $30 \mathrm{~s}$ and is repeated varying only the current imbalance $\delta I$ to collect a complete data set. The atom number difference between the left and right wells is then normalized by the sum $N_{L}+N_{R}$ yielding $\Delta N / N$ for each $\delta I$ measurement. Figure 4 shows the results from 35 individual $\delta I$ measurements collected randomly in time. From the distribution of the data we estimate that a measurement of $\Delta$ can be performed with a single shot sensitivity of $16 \mathrm{~Hz}$ where the dominant source of noise has been attributed to shot-to-shot condensate number fluctuation at the 3 percent level. This resolution is significantly better than that obtained with rf-spectroscopy which is typically limited by fluctuating background magnetic field noise at the $0.1 \mu \mathrm{T}$ level. Moreover, since the total potential asymmetry is measured, $\Delta$ is also sensitive to non-magnetic field sources such as gravity fields or light shifts. Although a magnetic field gradient is used above it is conceivable that the same measurements could be performed by tilting the chip or by introducing a gravitational field from a test mass. Using the $70 \mu \mathrm{m}$ separation we infer a single shot sensitivity to a gravity field of $\frac{\delta g}{g}=2 \times 10^{-4}$. At present this accuracy surpasses interferometric experiments with trapped atoms which appear to be limited due to phase decoherence for splitting times greater than a few milliseconds [4].

To provide a further understanding of this experiment a simple model is proposed whereby the double-well system is represented by two uncoupled 3-dimensional harmonic wells with ground states shown in Fig. 囵. Using the Thomas-Fermi approximation for a harmonic trap the chemical potential $\mu$ is given by,

$$
\mu(H z)=\frac{\bar{\omega}}{4 \pi}\left(\frac{15 N_{R} a}{a_{0}}\right)^{\frac{2}{5}}=\frac{\bar{\omega}}{4 \pi}\left(\frac{15 N_{L} a}{a_{0}}\right)^{\frac{2}{5}}+\Delta
$$

where $N_{L}$ and $N_{R}$ are the number of atoms in the left and right wells respectively, $\bar{\omega}$ is the geometric mean of the trap frequencies, $a$ is the atom scattering length and 

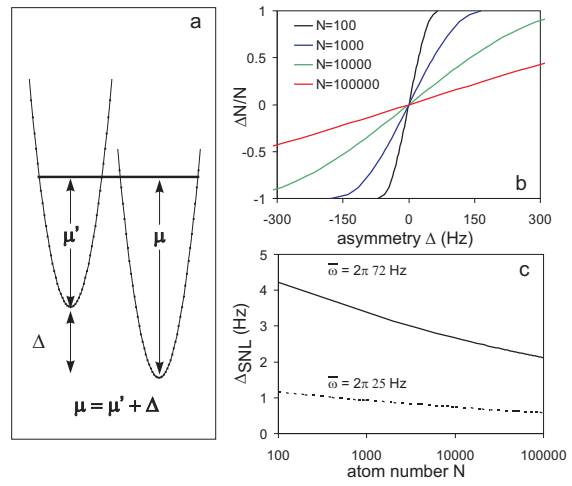

FIG. 5: . The asymmetric double well is represented by two identical harmonic traps offset by $\Delta$ with a ground state which obeys $\mu=\mu^{\prime}+\Delta$ (a). From this model we can extract the dependence of the fractional number difference on $\Delta$ for increasing atom number (b). In the standard quantum limit the sensitivity improves as $N^{-0.1}$ as shown for two trap conditions (c).

$a_{0}$ is the ground state harmonic oscillator length. Algebraic manipulation and a first order expansion about $\Delta=0$ yields the following relation for the fractional number difference between the left and right wells

$$
\frac{\Delta N}{N} \approx 1.65 \frac{\Delta}{c N^{\frac{2}{5}}} \quad c=\frac{\bar{\omega}}{4 \pi}\left(\frac{15 a}{a_{0}}\right)^{\frac{2}{5}}
$$

Inserting experimental parameters into Eq. 3 yields the

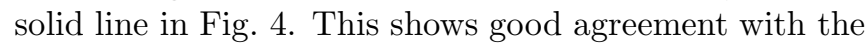
data considering the simplicity of the model. To investigate the limits of this technique $\frac{\Delta N}{N}$ was evaluated using Eq. 2 for several $\mathrm{N}$ values (Fig. [5). Decreasing atom number reduces $\mu$ such that smaller asymmetries yield larger $\frac{\Delta N}{N}$. However in the shot-noise limit the fractional number difference can be measured more accurately with increasing atom number as $\sigma_{\Delta N / N} \propto N^{-1 / 2}$. These two opposing behaviors are evident in Fig. 55 which shows that the shot-noise limit sensitivity depends only weakly on atom number $\left(N^{-0.1}\right)$. A more appropriate strategy to improve the measurement sensitivity is to decrease $\bar{\omega}$ thus relaxing the potential and lowering $\mu$ accordingly. Another approach which lends itself to the atom chip concept is to micro-fabricate multiple $(\times n)$ double-well potentials on a single chip thus gaining $\sqrt{n}$ enhancement per measurement cycle. Reasonable parameters for such a chip $\left(\mathrm{n}=100, \mathrm{~N}_{L, R}=2000\right.$ atoms/well and $\bar{\omega}=2 \pi \times 12 \mathrm{~Hz}$ ) yield a possible single shot sensitivity of $\Delta_{S N L} \approx 0.04 \mathrm{~Hz}$ or $\frac{\delta g}{g}=5 \times 10^{-7}$ for a well separation $\lambda=70 \mu \mathrm{m}$. While this sensitivity appears low compared to state-of-the-art gravimeters, it already falls into the range of interest for mineral prospecting while offering an atom chip sized package which is ultimately scalable 21].

In conclusion, a corrugated potential resulting from random magnetization inhomogeneities has been used to investigate the dynamical transfer of a condensate from a single to a double-well potential. Using radio frequency spectroscopy and absorption imaging techniques this potential was characterized. The sensitivity of the fractional number difference to a magnetic field gradient induced asymmetry was then investigated. A simple model predicting the sensitivity of this technique agrees well with observations and proves useful in motivating additional improvements to the gravity field sensitivity of future experiments.

This project is supported by the ARC Centre of Excellence for Quantum-Atom Optics and a Swinburne University Strategic Initiative grant.

* Electronic address: brhall@swin.edu.au

[1] A. Peters, K. Chung, and S. Chu, Nature 400, 849 (1999).

[2] T. L. Gustavson, P. Bouyer, and M. A. Kasevich, Phys. Rev. Lett. 78, 2046 (1997).

[3] Y. Wang et al., Phys. Rev. Lett. 94, 090405 (2005).

[4] T. Schumm et al., Nature Physics (London) 1, 57 (2005).

[5] S. Wildermuth et al., Nature (London) 435, 440 (2005).

[6] S. Whitlock et al., arXiv:physics/0605028 (2006).

[7] S. Wildermuth et al., arXiv:cond-mat/0512520 (2005).

[8] M. Jones et al., Phys. Rev. Lett. 91, 080401 (2003).

[9] C. Sinclair et al., Phys. Rev. A 72, 031603(R) (2005).

[10] D. Harber et al., Phys. Rev. A 72, 033610 (2005).

[11] G. Ferrari et al., arXiv:physics/0605018 (2006).

[12] B. V. Hall et al., J. Phys. B: At. Mol. Opt. Phys. 39, 27 (2006).

[13] J. Fortágh et al., Phys. Rev. A 66, 041604(R) (2002).

[14] J. Estève et al., Phys. Rev. A 70, 043629 (2004).

[15] R. Folman et al., Phys. Rev. Lett. 84, 4749 (2000).

[16] B. V. Hall et al., in Laser Spectroscopy XVII, edited by E. A. Hinds, A. Ferguson, and E. Riis (World Scientific, Singapore, 2005).

[17] J. Estève et al., Eur. Phys. J. D 35, 141 (2005).

[18] C. Henkel, S. Potting, and M. Wilkens, Appl. Phys. B 69, 379 (1999).

[19] C. Henkel et al., Appl. Phys. B 76, 173 (1999).

[20] E. Streed et al., Rev. Sci. Inst. 77, 023106 (2006).

[21] A. Peters, K. Chung, and S. Chu, Metrologia 38, 25 (1999). 